\title{
The uniformizing differential equation of the complex hyperbolic structure on the moduli space of marked cubic surface II
}

\author{
Takeshi SASAKI * Masaaki YOSHIDA ${ }^{\dagger}$
}

May 26, 2000

\begin{abstract}
The relation between the uniformizing equation of the complex hyperbolic structure on the moduli space of marked cubic surfaces and an AppellLauricella hypergeometric system in nine variables is clarified.
\end{abstract}

\section{Introduction}

In the previous paper $([3])$ we found the uniformizing equation which governs the developing map of the complex hyperbolic structure on the (4-dimensional) moduli space of marked cubic surfaces. Our equation is invariant under the action of the Weyl group of type $E_{6}$. Terasoma and Matsumoto are establishing a theory implying that the variation of Hodge structure of the cubic surfaces is essentially equivalent to that of a certain family of curves which are cyclic covers of the line branching at twelve points ([2]). This theory suggests that our uniformizing equation should be equivalent to a restriction of the Appell-Lauricella hypergeometric system $F_{D}$ in nine variables. In this paper, we carry out the computation and prove this observation.

\section{Configuration spaces $X(2, N)$}

Let $X(2, N)$ be the configuration space of $N$ (colored) points on the projective line $\mathrm{P}^{1}$ defined as

$$
X(2, N)=P G L_{2} \backslash\left\{\left(x_{1}, \ldots, x_{N}\right) \in\left(\mathrm{P}^{1}\right)^{N} \mid x_{i} \neq x_{j}(i \neq j)\right\} .
$$

By normalizing three points as $0,1, \infty$, the space $X(2, N)$ can be identified with the open affine set

$$
\prod_{j=1}^{n} x_{j}\left(1-x_{j}\right) \prod_{1 \leq i<j \leq n}\left(x_{i}-x_{j}\right) \neq 0
$$

*Department of Mathematics, Kobe University, Kobe 657 Japan

${ }^{\dagger}$ Department of Mathematics, Kyushu University 01, Fukuoka 810 Japan 
in the affine space coordinatized by $\left(x_{1}, \ldots, x_{n}\right)$, where $n+3=N$. On this configuration space lives the Appell-Lauricella hypergeometric system $E_{D}$, which we review in the next section. When $n=1, X(2,4)$ is isomorphic to $\mathbf{C}-\{0,1\}$.

\section{Appell-Lauricella hypergeometric system $E_{D}$}

For coordinates $x_{1}, \ldots, x_{n}$, put $D_{i}:=x_{i} \partial / \partial x_{i}$. The system

$$
D_{i} D_{j} u=\sum_{k=1}^{n} p_{i j}^{k} D_{k} u+p_{i j}^{0} u \quad(i, j=1, \ldots, n),
$$

with parameters $(a, b, c)=\left(a, b_{1}, \ldots, b_{n}, c\right)$, where

$$
\begin{aligned}
p_{i j}^{k} & =p_{j i}^{k}, \quad p_{i j}^{0}=p_{j i}^{0}, \quad 1 \leq i, j, k \leq n ; \quad p_{i j}^{0} p_{i j}^{k}=0, \quad i \neq j \neq k \neq i \\
p_{i j}^{i} & =b_{j} \frac{x_{j}}{x_{i}-x_{j}}, \quad i \neq j, \quad p_{i i}^{0}=a b_{i} \frac{x_{i}}{1-x_{i}}, \\
p_{i i}^{k} & =b_{i}\left(\frac{x_{i}}{1-x_{i}}-\frac{x_{i}}{x_{k}-x_{i}}\right), \quad i \neq k \\
p_{i i}^{i} & =-\sum_{k \neq i} b_{k} \frac{x_{k}}{x_{i}-x_{k}}+\frac{\left(a+b_{i}\right) x_{i}-c+1}{1-x_{i}}
\end{aligned}
$$

is called the Appell-Lauricella hypergeometric system of type $D$ and is denoted by $E_{D}^{n}\left(a, b_{1}, \ldots, b_{n}, c\right)$. The Appell-Lauricella hypergeometric series

$$
\begin{aligned}
& F_{D}\left(a ; b_{1}, \ldots, b_{n} ; c \mid x^{1}, \ldots, x^{n}\right)= \\
& \quad \sum_{m_{1}, \ldots, m_{n}=0}^{\infty} \frac{\left(a, m_{1}+\cdots+m_{n}\right)\left(b_{1}, m_{1}\right) \cdots\left(b_{n}, m_{n}\right)}{\left(c, m_{1}+\cdots+m_{n}\right) m_{1} ! \cdots m_{n} !}\left(x^{1}\right)^{m_{1}} \cdots\left(x^{n}\right)^{m_{n}}
\end{aligned}
$$

where $(a, m)=a(a+1) \cdots(a+m-1)$, solves this system around the origin $([\mathbb{1}], \mathbb{1}])$. This system has singularities along the divisor defined by

$$
\prod_{j=1}^{n} x_{j}\left(1-x_{j}\right) \prod_{1 \leq i<j \leq n}\left(x_{i}-x_{j}\right)=0
$$

and at infinity, that is, this system is regular in the configuration space $X(2, n+3)$. The rank (the dimension of the space of local solutions at a(ny) regular point) is $n+1$. When $n=1, E_{D}^{1}(a, b, c)$ is the Gauss hypergeometric equation.

Note in general that if a system of the above form is of rank $n+1$ then the coefficients $p_{i j}^{0}$ can be expressed in terms of $p_{p q}^{r}(1 \leq p, q, r \leq n)$ and their derivatives; so we often describe a system by presenting $p_{i j}^{k}$ only. 


\section{The pull-back of $E_{D}$ under an embedding of $X(2, n+3)$ into $X(2,2 n+4)$}

Let us embed (a Zariski open subset of) the space $X(2, n+3)$ into $X(2,2 n+4)$ as

$$
\begin{aligned}
\iota: X(2, n+3) & \ni\left(0, \infty, 1, x_{1}, \ldots, x_{n}\right) \\
& \longmapsto\left(0, \infty, 1, x_{1}, \ldots, x_{n},-x_{1}, \ldots,-x_{n},-1\right) \in X(2,2 n+4) .
\end{aligned}
$$

The space $X(2,2 n+4)$ is of dimension $2 n+1$ and the Appell-Lauricella hypergeometric system $E_{D}^{2 n+1}$ is of rank $2 n+2$.

For a solution $u=u\left(x_{1}, \ldots, x_{2 n+1}\right)$ of $E_{D}^{2 n+1}=E_{D}^{2 n+1}\left(a, b_{1}, \ldots, b_{2 n+1}, c\right)$, put

$$
v\left(x_{1}, \ldots, x_{n}\right):=u\left(x_{1}, \ldots, x_{n},-x_{1}, \ldots,-x_{n},-1\right) .
$$

For generic parameters $(a, b, c)$, the system satisfied by the pull-back $v$ of $u$ under $\iota$ would be of rank $2 n+2$. We study whether the rank of this system may be of rank $n+1$. We have

$$
\begin{aligned}
D_{i} v & =\left(D_{i}+D_{i+n}\right) u, \quad 1 \leq i \leq n \\
D_{i} D_{j} v & =\left(D_{i} D_{j}+D_{i} D_{j+n}+D_{i+n} D_{j}+D_{i+n} D_{j+n}\right) u \\
& =\sum_{k=0}^{2 n+1}\left(p_{i, j}^{k}+p_{i, j+n}^{k}+p_{i+n, j}^{k}+p_{i+n, j+n}^{k}\right) D_{k} u
\end{aligned}
$$

where $D_{0} u:=u$, and the right hand-sides denote the values restricted to

$$
x_{n+1}=-x_{1}, \ldots, x_{2 n}=-x_{n}, \quad x_{2 n+1}=-1 .
$$

For $1 \leq i \neq j \leq n$, we have

$$
\begin{aligned}
D_{i} D_{j} v & =\sum_{k=1}^{2 n}\left(p_{i, j}^{k}+p_{i, j+n}^{k}+p_{i+n, j}^{k}+p_{i+n, j+n}^{k}\right) D_{k} u \\
& =\left(p_{i, j}^{i}+p_{i, j+n}^{i}\right) D_{i} u+\left(p_{i+n, j}^{i+n}+p_{i+n, j+n}^{i+n}\right) D_{i+n} u \\
& +\left(p_{i, j}^{j}+p_{i+n, j}^{j}\right) D_{j} u+\left(p_{i, j+n}^{j+n}+p_{i+n, j+n}^{j+n}\right) D_{j+n} u
\end{aligned}
$$

and

$$
\begin{aligned}
p_{i, j}^{i}+p_{i, j+n}^{i} & =b_{j} \frac{x_{j}}{x_{i}-x_{j}}+b_{j+n} \frac{x_{j+n}}{x_{i}-x_{j+n}} \\
& =b_{j} \frac{x_{j}}{x_{i}-x_{j}}+b_{j+n} \frac{-x_{j}}{x_{i}+x_{j}}, \\
p_{i+n, j}^{i+n}+p_{i+n, j+n}^{i+n} & =b_{j} \frac{x_{j}}{x_{i+n}-x_{j}}+b_{j+n} \frac{x_{j+n}}{x_{i+n}-x_{j+n}} \\
& =b_{j} \frac{x_{j}}{-x_{i}-x_{j}}+b_{j+n} \frac{-x_{j}}{-x_{i}+x_{j}} .
\end{aligned}
$$


When $i=j$, we have

$$
\begin{aligned}
D_{i}^{2} v & =\sum_{k=0}^{2 n+1}\left(p_{i, i}^{k}+2 p_{i, i+n}^{k}+p_{i+n, i+n}^{k}\right) D_{k} u \\
& =\left(p_{i, i}^{i}+2 p_{i, i+n}^{i}+p_{i+n, i+n}^{i}\right) D_{i} u+\left(p_{i, i}^{i+n}+2 p_{i, i+n}^{i+n}+p_{i+n, i+n}^{i+n}\right) D_{i+n} u \\
& +\sum_{1 \leq k \neq i \leq n}\left[\left(p_{i, i}^{k}+p_{i+n, i+n}^{k}\right) D_{k} u+\left(p_{i, i}^{k+n}+p_{i+n, i+n}^{k+n}\right) D_{k+n} u\right] \\
& +\left(p_{i, i}^{2 n+1}+p_{i+n, i+n}^{2 n+1}\right) D_{2 n+1} u+\left(p_{i, i}^{0}+p_{i+n, i+n}^{0}\right) u,
\end{aligned}
$$

and

$$
\begin{aligned}
& p_{i, i}^{2 n+1}=b_{i}\left(\frac{x_{i}}{1-x_{i}}-\frac{x_{i}}{x_{2 n+1}-x_{i}}\right)=b_{i}\left(\frac{x_{i}}{1-x_{i}}-\frac{x_{i}}{-1-x_{i}}\right) \\
& p_{i+n, i+n}^{2 n+1}=b_{i+n}\left(\frac{x_{i+n}}{1-x_{i+n}}-\frac{x_{i+n}}{x_{2 n+1}-x_{i+n}}\right)=b_{i+n}\left(\frac{-x_{i}}{1+x_{i}}-\frac{-x_{i}}{-1+x_{i}}\right) \text {, } \\
& p_{i, i}^{k}+p_{i+n, i+n}^{k}=b_{i}\left(\frac{x_{i}}{1-x_{i}}-\frac{x_{i}}{x_{k}-x_{i}}\right)+b_{i+n}\left(\frac{-x_{i}}{1+x_{i}}-\frac{-x_{i}}{x_{k}+x_{i}}\right), \\
& p_{i, i}^{k+n}+p_{i+n, i+n}^{k+n}=b_{i}\left(\frac{x_{i}}{1-x_{i}}-\frac{x_{i}}{-x_{k}-x_{i}}\right)+b_{i+n}\left(\frac{-x_{i}}{1+x_{i}}-\frac{-x_{i}}{-x_{k}+x_{i}}\right), \\
& p_{i, i}^{i}+2 p_{i, i+n}^{i}+p_{i+n, i+n}^{i}=-\sum_{1 \leq k \neq i \leq 2 n+1} b_{k} \frac{x_{k}}{x_{i}-x_{k}}+\frac{1}{1-x_{i}}\left\{\left(a+b_{i}\right) x_{i}-c+1\right\} \\
& +2 b_{i+n} \frac{x_{i+n}}{x_{i}-x_{i+n}}+b_{i+n}\left(\frac{x_{i+n}}{1-x_{i+n}}-\frac{x_{i+n}}{x_{i}-x_{i+n}}\right) \\
& =-\sum_{1 \leq k \neq i \leq n}\left(b_{k} \frac{x_{k}}{x_{i}-x_{k}}+b_{k+n} \frac{-x_{k}}{x_{i}+x_{k}}\right) \\
& +\frac{\left(a+b_{i}+b_{i+n}\right) x_{i}^{2}+\left(a-c-b_{2 n+1}+1+b_{i}-b_{i+n}\right) x_{i}+b_{2 n+1}-c+1}{\left(1-x_{i}\right)\left(1+x_{i}\right)}, \\
& p_{i, i}^{i+n}+2 p_{i, i+n}^{i+n}+p_{i+n, i+n}^{i+n}=b_{1}\left\{\frac{x_{i}}{1-x_{i}}-\frac{x_{i}}{-x_{i}-x_{i}}\right\}+2 b_{i} \frac{x_{i}}{-x_{i}-x_{i}} \\
& +\frac{1}{1+x_{i}}\left\{\left(a+b_{i+n}\right)\left(-x_{i}\right)-c+1 \frac{-1}{x_{i}}\right\}-\sum_{1 \leq k \neq i+n \leq 2 n+1} b_{k} \frac{x_{k}}{x_{i+n}-x_{k}} \\
& =-\sum_{1 \leq k \neq i \leq n}\left(b_{k} \frac{x_{k}}{-x_{i}-x_{k}}+b_{k+n} \frac{-x_{k}}{-x_{i}+x_{k}}\right) \\
& +\frac{\left(a+b_{i}+b_{i+n}\right) x_{i}^{2}+\left(-a+c+b_{2 n+1}-1+b_{i}-b_{i+n}\right) x_{i}+b_{2 n+1}-c+1}{\left(1-x_{i}\right)\left(1+x_{i}\right)} .
\end{aligned}
$$

In order the system for $v$ to be of rank $n+1, D_{i} D_{j} v$ must be linearly related with $D_{k} v$ and $v$. We have

$$
p_{i, i}^{2 n+1}+p_{i+n, i+n}^{2 n+1}=0 \quad \text { and } \quad p_{i, j}^{k}+p_{i+n, j+n}^{k}=p_{i, j}^{k+n}+p_{i+n, j+n}^{k+n} \quad(1 \leq i, j \leq n),
$$


if and only if $b_{i}=b_{i+n}(1 \leq i \leq n)$. Assuming these, we have

$$
p_{i, i}^{i}+2 p_{i, i+n}^{i}+p_{i+n, i+n}^{i}=p_{i, i}^{i+n}+2 p_{i, i+n}^{i+n}+p_{i+n, i+n}^{i+n} \quad(1 \leq i \leq n),
$$

if and only if $a-c-b_{2 n+1}+1=0$.

Proposition 1 The pull-back, under the embedding $\iota: X(2, n+3) \rightarrow X(2,2 n+$ 4) of a (ny) non-zero solution of the hypergeometic system $E_{D}^{2 n+1}\left(a, b_{1}, \ldots, b_{2 n+1}, c\right)$ satisfies a system of rank $n+1$ if and only if

$$
b_{i}=b_{i+n}, \quad(1 \leq i \leq n) \quad \text { and } \quad a-c-b_{2 n+1}+1=0 .
$$

This system of rank $n+1$ on $X(2, n+3)$, which will be called $\mathcal{E}\left(a, b_{1}, \ldots, b_{n}, c\right)$, is given by

$$
D_{i} D_{j} v=\sum_{k=1}^{n} q_{i j}^{k} D_{k} v+q_{i j}^{0} v \quad(1 \leq i, j \leq n),
$$

with parameters $\left(a, b_{1}, \ldots, b_{n}, c\right)$, where

$$
\begin{aligned}
q_{i j}^{k} & =q_{j i}^{k}, \quad q_{i j}^{0}=q_{j i}^{0}, \quad 1 \leq i, j, k \leq n ; \quad q_{i j}^{0}=q_{i j}^{k}=0, \quad i \neq j \neq k \neq i \\
q_{i j}^{i} & =p_{i, j}^{i}+p_{i, j+n}^{i}=2 b_{j} \frac{x_{j}^{2}}{x_{i}^{2}-x_{j}^{2}}, \quad i \neq j \\
q_{i i}^{0} & =p_{i, i}^{0}+p_{i+n, i+n}^{0}=a\left(2 b_{i}\right) \frac{x_{i}^{2}}{1-x_{i}^{2}}, \\
q_{i i}^{k} & =p_{i, i}^{k}+p_{i+n, i+n}^{k}=2 b_{i}\left(\frac{x_{i}^{2}}{1-x_{i}^{2}}-\frac{x_{i}^{2}}{x_{k}^{2}-x_{i}^{2}}\right), \quad i \neq k \\
q_{i i}^{i} & =p_{i, i}^{i}+2 p_{i, i+n}^{i}+p_{i+n, i+n}^{i} \\
& =-\sum_{k \neq i} 2 b_{k} \frac{x_{k}^{2}}{x_{i}^{2}-x_{k}^{2}}+\frac{\left(a+2 b_{i}\right) x_{i}^{2}+a-2 c+2}{1-x_{i}^{2}} .
\end{aligned}
$$

The system has regular singularities along

$$
\prod_{i=1}^{n} x_{i}\left(1-x_{i}\right)\left(1+x_{i}\right) \prod_{1 \leq i<j \leq n}\left(x_{i}-x_{j}\right)\left(x_{i}+x_{j}\right)=0
$$

and at infinity.

Rewriting this system in the form $\partial^{2} v / \partial x_{i} \partial x_{j}=\cdots$, we get

Corollary 1 This system is non-singular along $\left\{x_{j}=0\right\}$ if and only if

$$
\left(\left.q_{i i}^{i}\right|_{x_{i}=0}=\right) \quad 2 \sum_{1 \leq k(\neq j) \leq n} b_{k}+a-2 c+2=0 .
$$

We need not check that this system is of rank $n+1$, because of the following fact. Note that if we introduce the variables $y_{j}=x_{j}^{2}$, then we have

$$
D_{j}^{x}=2 D_{j}^{y}, \quad \text { where } D_{j}^{x}=x_{j} \partial / \partial x_{j}, D_{j}^{y}=y_{j} \partial / \partial y_{j} .
$$

Comparing the coefficients of $\mathcal{E}(a, b, c)$ and those of $E_{D}^{n}(a, b, c)$, we have 
Corollary 2 The Appell-Lauricella system $E_{D}^{n}\left(a / 2, b_{1}, \ldots, b_{n}, c-a / 2\right)$ in y-variables is transformed into $\mathcal{E}(a, b, c)$ in $x$-variables by the change $y_{j}=x_{j}^{2}$.

Remark 1 The following integral representation of a solution of $E_{D}^{2 n+1}(a, b, c)$

$$
\int t^{a-1}(1-t)^{c-a-1}\left(1-x_{1}\right)^{-b_{1}} \cdots\left(1-x_{2 n}\right)^{-b_{2 n}}\left(1-x_{2 n+1}\right)^{-b_{2 n+1}} d t
$$

supports Proposition 1 and Corollary 2.

\section{Systems invariant under the involution \#}

Conisder the involution

$$
\#:\left(x_{1}, \ldots, x_{n}\right) \longmapsto\left(1 / x_{1}, \ldots 1 / x_{n}\right)
$$

on $X(2, n+3)$ and let \# takes the system $E(a, b, c)=E_{D}^{n}\left(a, b_{1}, \ldots, b_{n}, c\right)$ into a system

$$
{ }^{\#} E(a, b, c): D_{i} D_{j} u=\sum_{k=1}^{n} \pi_{i j}^{k} D_{k} u+\pi_{i j}^{0} u, \quad(1 \leq i, j \leq n) .
$$

Since the change of variables $x_{i} \rightarrow 1 / x_{i}$ induces the change of the derivations $D_{i} \rightarrow$ $-D_{i}$, we can easily see the coefficients:

$$
\begin{aligned}
\pi_{i j}^{k} & =\pi_{j i}^{k}, \quad \pi_{i j}^{0}=\pi_{j i}^{0}, \quad 1 \leq i, j, k \leq n ; \quad \pi_{i j}^{0}=\pi_{i j}^{k}=0, \quad i \neq j \neq k \neq i, \\
\pi_{i j}^{i} & =b_{j} \frac{x_{i}}{x_{i}-x_{j}}, \quad i \neq j, \quad \pi_{i i}^{0}=a b_{i} \frac{1}{1-x_{i}}, \\
\pi_{i i}^{k} & =b_{i}\left(\frac{1}{1-x_{i}}-\frac{x_{k}}{x_{k}-x_{i}}\right), \quad i \neq k \\
\pi_{i i}^{i} & =-\sum_{k \neq i} b_{k} \frac{x_{i}}{x_{i}-x_{k}}+\frac{(-c+1) x_{i}+a+b_{i}}{1-x_{i}} .
\end{aligned}
$$

In particular $E(a, b, c)$ is not invariant under the involution \# for any choice of parameters not simultaneously zero.

Let us transform the systems $E$ and ${ }^{\#} E$ into the normal forms defined below. We change the unknown $v$ of the system $E(a, b, c)$ as $v=\rho w$, where $\rho$ is a non-zero function. If we write the new system as

$$
{ }^{N} E(a, b, c): D_{i} D_{j} w=\sum_{k=1}^{n} P_{i j}^{k} D_{k} w+P_{i j}^{0} w, \quad(1 \leq i, j \leq n)
$$

then the coefficients $P_{i j}^{k}$ are given by

$$
P_{i j}^{k}=p_{i j}^{k}-\frac{D_{i} \rho}{\rho} \delta_{j}^{k}-\frac{D_{j} \rho}{\rho} \delta_{i}^{k},
$$


where $\delta$ denotes the Kronecker symbol. Now choose $\rho$ so that the system ${ }^{N} E$ be of normal form, which means by definition,

$$
\sum_{k=1}^{n} P_{k j}^{k}=0, \quad(1 \leq j \leq n)
$$

that is,

$$
\sum_{k=1}^{n} p_{k j}^{k}-(n+1) \frac{D_{j} \rho}{\rho}=0 ; \quad(1 \leq j \leq n)
$$

it is known that if the given system $E$ is integrable then there is a non-zero function $\rho$ solving the above first order system of differential equations. Thus we have

$$
P_{i j}^{k}=p_{i j}^{k}-\frac{\delta_{j}^{k}}{n+1} p_{i}-\frac{\delta_{i}^{k}}{n+1} p_{j},
$$

where

$$
p_{j}=\sum_{k=1}^{n} p_{k j}^{k}=\sum_{k \neq j} p_{k j}^{k}+p_{j j}^{j}=\sum_{k \neq j} \frac{b_{j} x_{j}+b_{k} x_{k}}{x_{k}-x_{j}}+\frac{\left(a+b_{j}\right) x_{j}-c+1}{1-x_{j}} .
$$

The coefficients of the normal form ${ }^{N} E(a, b, c)$ are given by

$$
\begin{aligned}
P_{i i}^{k} & =p_{i i}^{k}=\frac{b_{i} x_{i}\left(x_{k}-1\right)}{\left(1-x_{i}\right)\left(x_{k}-x_{i}\right)}, \quad i \neq k, \\
P_{i j}^{i} & =p_{i j}^{i}-\frac{p_{j}}{n+1}=\frac{b_{j} x_{j}}{x_{i}-x_{j}}-\frac{p_{j}}{n+1}, \quad i \neq j \\
P_{i i}^{i} & =p_{i i}^{i}-2 \frac{p_{j}}{n+1} .
\end{aligned}
$$

We next find the normal form

$$
{ }^{N \#} E(a, b, c): D_{i} D_{j} w=\sum_{k=1}^{n} \Pi_{i j}^{k} D_{k} w+\Pi_{i j}^{0} w, \quad(1 \leq i, j \leq n)
$$

of ${ }^{\#} E(a, b, c)$. Its coefficients are given by

$$
\Pi_{i j}^{k}=\pi_{i j}^{k}-\frac{\delta_{j}^{k}}{n+1} \pi_{i}-\frac{\delta_{i}^{k}}{n+1} \pi_{j},
$$

where

$$
\pi_{j}=\sum_{k=1}^{n} \pi_{k j}^{k}=\sum_{k \neq j} \pi_{k j}^{k}+\pi_{j j}^{j}=\sum_{k \neq j} \frac{b_{j} x_{k}+b_{k} x_{j}}{x_{k}-x_{j}}+\frac{(-c+1) x_{j}+a+b_{j}}{1-x_{j}} .
$$

Thus we have

$$
\begin{aligned}
\Pi_{i i}^{k} & =\pi_{i i}^{k}=\frac{b_{i} x_{i}\left(x_{k}-1\right)}{\left(1-x_{i}\right)\left(x_{k}-x_{i}\right)}, \quad i \neq k, \\
\Pi_{i j}^{i} & =\pi_{i j}^{i}-\frac{\pi_{j}}{n+1}=\frac{b_{j} x_{i}}{x_{i}-x_{j}}-\frac{\pi_{j}}{n+1}, \quad i \neq j \\
\Pi_{i i}^{i} & =\pi_{i i}^{i}-2 \frac{\pi_{j}}{n+1} .
\end{aligned}
$$


Compare the coefficients and recall an elementary fact that a rational function

$$
\sum_{j} \frac{\alpha_{j} x+\beta_{j}}{x-t_{j}}
$$

in $x$ vanishes identically if and only if $\alpha_{j} t_{j} \sum_{j}+\beta_{j}=0$ and $\sum_{j} \alpha_{j}=0$. Then we get

Proposition 2 The system ${ }^{N} E(a, b, c)$ coincides with the system ${ }^{N \#} E(a, b, c)$ if and only if

$$
b_{1}=\cdots=b_{n}(=b), \quad-n b+a+c-1=0 .
$$

Let us summarize the above computaion. We got a system $\mathcal{E}(a, b, c)=\mathcal{E}\left(a, b_{1}, \ldots, b_{n}, c\right)$ of rank $n+1$ on $X(2, n+3)$, which was the pull-back under $\iota: X(2, n+3) \rightarrow$ $X(2,2 n+4)$ of the hypergeometric system $E_{D}^{2 n+1}\left(a, b_{1}, \ldots, b_{2 n+1}, c\right)$, with

$$
b_{n+1}=b_{1}, \ldots, b_{2 n}=b_{n}, b_{2 n+1}=a-c+1 .
$$

The normal forms of $\mathcal{E}(a, b, c)$ and its pull-back coincide if and only if

$$
b_{1}=\cdots=b_{n}(=b), \quad-(2 n+1) b+a+c-1 .
$$

These conditions lead to

$$
a=(n+1) b, \quad c=n b+1 .
$$

If moreover $\mathcal{E}$ is regular along the divisors $\left\{x_{j}=0\right\}$, then we have $b=1 /(n-1)$.

\section{The double covering $f: X(2,7) \rightarrow X(3,6)$}

Let $X(3,6)$ be the configuration space of colored six points in general position in the projective plane

$$
X(3,6)=G L_{3} \backslash\{z \in M(3,6) \mid \text { no 3-minor vanishes }\} /\left(\mathbf{C}^{\times}\right)^{6} .
$$

We define a rational map $f$ from $X(2,7)$ to $X(3,6)$. We start from a system of seven points on the line representing a point of $X(2,7)$. We regard the line, carrying the seven points, as a non-singular conic in the plane. The five points represented by the last five points, and the intersection point of the tangent lines (to the conic) at the first and the second points define a system of six points on the plane, representing a point of $X(3,6)$. Let us express this map $f$ in terms of coordinates. We normalize the system of seven points to be

$$
x=\left(0, \infty, 1, x_{1}, \ldots, x_{4}\right) \in X(2,7) .
$$

If the conic is given by $t_{1}^{2}-t_{0} t_{2}=0$ in the plane coordinatized by $t_{0}: t_{1}: t_{2}$, the seven points are represented by the seven columns

$\begin{array}{ccccccc}1 & 0 & 1 & 1 & 1 & 1 & 1 \\ 0 & 0 & 1 & x_{1} & x_{2} & x_{3} & x_{4} \\ 0 & 1 & 1 & x_{1}^{2} & x_{2}^{2} & x_{3}^{2} & x_{4}^{2}\end{array}$.


Since the tangents of the conic at the first two points are $t_{2}=0$ and $t_{0}=0$, the intersection is given by $0: 1: 0$; so the point $f(x)$ is represented by

$$
\left(\begin{array}{cccccc}
0 & 1 & 1 & 1 & 1 & 1 \\
1 & 1 & x_{1} & x_{2} & x_{3} & x_{4} \\
0 & 1 & x_{1}^{2} & x_{2}^{2} & x_{3}^{2} & x_{4}^{2}
\end{array}\right) \in X(3,6)
$$

Normalizing the above $3 \times 6$-matrix into

$$
\left(\begin{array}{cccccc}
1 & 0 & 0 & 1 & 1 & 1 \\
0 & 1 & 0 & 1 & z_{1} & z_{2} \\
0 & 0 & 1 & 1 & z_{3} & z_{4}
\end{array}\right)
$$

we can readily show

Proposition 3 The map

$$
f: X(2,7) \ni x=\left(x_{1}, \ldots, x_{4}\right) \longmapsto z=\left(z_{1}, \ldots, z_{4}\right) \in X(3,6)
$$

defined in this section is given by

$$
\begin{aligned}
& z_{1}=\frac{\left(x_{3}+x_{1}\right)\left(x_{2}-1\right)}{\left(x_{3}-1\right)\left(x_{2}+x_{1}\right)}, \quad z_{2}=\frac{\left(x_{4}+x_{1}\right)\left(x_{2}-1\right)}{\left(x_{4}-1\right)\left(x_{2}+x_{1}\right)}, \\
& z_{3}=\frac{\left(x_{3}+1\right)\left(x_{2}-x_{1}\right)}{\left(x_{3}-x_{1}\right)\left(x_{2}+1\right)}, \quad z_{4}=\frac{\left(x_{4}+1\right)\left(x_{2}-x_{1}\right)}{\left(x_{4}-x_{1}\right)\left(x_{2}+1\right)} .
\end{aligned}
$$

Remark 2 1. The Jacobian of $f$ is given by

$$
\frac{\left(x_{1}-1\right)\left(-x_{2}+x_{1}\right)\left(x_{2}-x_{4}\right)\left(x_{3}-x_{4}\right)\left(x_{3}-x_{2}\right)\left(x_{1}+1\right)^{3}\left(x_{2}-1\right)}{\left(x_{3}-x_{1}\right)^{2}\left(x_{2}+1\right)^{3}\left(x_{4}-1\right)^{2}\left(x_{2}+x_{1}\right)^{3}\left(x_{3}-1\right)^{2}\left(-x_{4}+x_{1}\right)^{2}} .
$$

2. Put

$$
\begin{aligned}
D_{1} & =z_{1} z_{4}-z_{2} z_{3}, \quad D_{2}=z_{1} z_{4}-z_{2} z_{3}-z_{4}+z_{2}+z_{3}-z_{1}, \\
Q & =-z_{2} z_{3} z_{1}-z_{2} z_{3} z_{4}+z_{2} z_{3}+z_{1} z_{4} z_{2}+z_{1} z_{4} z_{3}-z_{1} z_{4} .
\end{aligned}
$$

Then the singular locus of the system found in 3 is defined by

$$
D=\prod_{j=1}^{4} z_{j}\left(1-z_{j}\right) \cdot\left(z_{1}-z_{2}\right)\left(z_{1}-z_{3}\right)\left(z_{2}-z_{4}\right)\left(z_{3}-z_{4}\right) D_{1} D_{2} Q
$$

and $f^{*}(D)$ is given by

$$
\begin{aligned}
& -\left(x_{3}+x_{1}\right)\left(x_{2}-1\right)^{5}\left(x_{4}+x_{1}\right)\left(x_{3}+1\right)\left(-x_{2}+x_{1}\right)^{5}\left(x_{4}+1\right)\left(x_{1}+1\right)^{13} \\
& \times\left(x_{3}-x_{2}\right)^{5}\left(x_{2}-x_{4}\right)^{5}\left(x_{3}-x_{4}\right)^{5}\left(x_{1}-1\right)^{5}\left(x_{3}+x_{2}\right)\left(x_{2}+x_{4}\right)\left(x_{3}+x_{4}\right) \\
& \times\left(x_{3}-1\right)^{-7}\left(x_{2}+x_{1}\right)^{-11}\left(x_{4}-1\right)^{-7}\left(x_{3}-x_{1}\right)^{-7}\left(x_{2}+1\right)^{-11}\left(-x_{4}+x_{1}\right)^{-7}
\end{aligned}
$$


Proposition 4 The map $f$ is invariant under the involution $\#: x_{i} \rightarrow 1 / x_{i}$. Moreover $f$ is a two-to-one map.

Sketch of the proof. For given $\left(z_{1}, \ldots, z_{4}\right)$, we solve $x_{1}, \ldots, x_{4}$. We can see that $x_{2}$ must be a solution of the quadratic equation

$$
C_{2} Z^{2}+C_{1} Z+C_{0}=0
$$

where

$$
\begin{aligned}
C_{0} & =C_{2}=-\left(-z_{2} z_{4}+z_{3} z_{2} z_{4}-z_{3}+z_{1} z_{3}+z_{4}-z_{1} z_{3} z_{4}\right) \\
& \times\left(z_{3}+z_{2}-z_{4}-z_{1}-z_{1} z_{3} z_{4}+z_{1} z_{3} z_{2}-z_{2} z_{4} z_{1}+z_{3} z_{2} z_{4}-2 z_{2} z_{3}+2 z_{1} z_{4}\right), \\
C_{1} & =-6 z_{1} z_{3} z_{4}+4 z_{2}^{2} z_{4} z_{1}-4 z_{2}^{2} z_{1} z_{3}+4 z_{3}^{2} z_{2}^{2} z_{1}+4 z_{4}^{2} z_{1}^{2} z_{2}-4 z_{1}^{2} z_{2} z_{4}+4 z_{1}^{2} z_{3} z_{2} \\
& -2 z_{1} z_{3} z_{2}-2 z_{2} z_{4} z_{1}-6 z_{3} z_{2} z_{4}-6 z_{2} z_{4}^{2} z_{1} z_{3}+16 z_{2} z_{4} z_{1} z_{3}-2 z_{1}^{2} z_{3} z_{4}^{2} z_{2} \\
& -2 z_{3}^{2} z_{1} z_{2}-2 z_{1}^{2} z_{3}^{2} z_{2}-2 z_{4}^{2} z_{2} z_{1}-2 z_{1}^{2} z_{3} z_{4}+2 z_{1}^{2} z_{3}^{2} z_{4}-2 z_{3}^{2} z_{2}^{2} z_{4}^{2}-2 z_{3} z_{2}^{2} z_{4} \\
& -2 z_{2}^{2} z_{4}^{2} z_{1}+2 z_{2}^{2} z_{4}^{2} z_{3}+4 z_{3}^{2} z_{2}^{2} z_{4}-2 z_{1}^{2} z_{3}^{2} z_{4}^{2}+4 z_{1}^{2} z_{3} z_{4}^{2}-2 z_{3}^{2}-2 z_{4}^{2} \\
& -4 z_{3}^{2} z_{2}^{2}+2 z_{1} z_{3}-2 z_{2} z_{3}+4 z_{4} z_{3}+4 z_{4} z_{1}^{2}-4 z_{4}^{2} z_{1}^{2}+2 z_{2} z_{4}+4 z_{1} z_{4}^{2}-2 z_{1} z_{4} \\
& +2 z_{1} z_{3}^{2}-2 z_{1}^{2} z_{3}+4 z_{3}^{2} z_{2}-2 z_{2}^{2} z_{4}+2 z_{2} z_{4}^{2}+2 z_{1}^{2} z_{3}^{2} z_{4} z_{2}+4 z_{3} z_{2}^{2}+2 z_{3} z_{2}^{2} z_{4}^{2} z_{1} \\
& -2 z_{3}^{2} z_{2}^{2} z_{4} z_{1}-2 z_{2}^{2} z_{4} z_{1} z_{3}+4 z_{1} z_{3}^{2} z_{4}^{2} z_{2}-2 z_{1}^{2} z_{3} z_{2} z_{4}-6 z_{1} z_{3}^{2} z_{2} z_{4} .
\end{aligned}
$$

The discriminant of the quadratic equation with respect to $Z$ is given by

$$
16\left(z_{1}-1\right)\left(z_{2}-1\right)\left(z_{3}-1\right)\left(z_{4}-1\right)\left(z_{1}-z_{2}\right)\left(z_{3}-z_{4}\right) D_{2} Q .
$$

Other ones $x_{1}$ and $x_{3}$ can be expressed as rational functions in $x_{2}$ and $z$ :

$$
\begin{aligned}
x_{1} & =-x_{2}\left(-z_{1} z_{3}+z_{1} z_{3} z_{2}+z_{2} z_{4}-z_{2} z_{4} z_{1}-z_{2}+z_{1}\right) /\left(-z_{3} x_{2}+z_{3}+z_{2}+z_{4} x_{2}\right. \\
& -z_{4}-z_{1}-z_{1} z_{3} z_{4}-z_{1} z_{3} z_{4} x_{2}+z_{1} z_{3} z_{2}-z_{2} z_{4} z_{1}+z_{3} z_{2} z_{4} x_{2}+z_{3} z_{2} z_{4} \\
& \left.+x_{2} z_{1} z_{3}-2 z_{2} z_{3}-x_{2} z_{2} z_{4}+2 z_{1} z_{4}\right), \\
x_{3} & =-x_{2}\left(1-2 z_{1}+z_{1} z_{3}-x_{2}+x_{2} z_{1} z_{3}\right)\left(z_{3}+z_{2}-z_{4}-z_{1}-z_{1} z_{3} z_{4}+z_{1} z_{3} z_{2}\right. \\
& \left.-z_{2} z_{4} z_{1}+z_{3} z_{2} z_{4}-2 z_{2} z_{3}+2 z_{1} z_{4}\right) /\left(2 x_{2} z_{3}^{2} z_{1} z_{4}+z_{3}+z_{2}-z_{4}-z_{1}\right. \\
& -6 z_{1} z_{3} z_{4}+2 z_{1} z_{3} z_{2}-z_{2} z_{4} z_{1}+z_{3} z_{2} z_{4}+2 z_{2} z_{4} z_{1} z_{3}-4 z_{3}^{2} z_{1} z_{2} \\
& +z_{1}^{2} z_{3}^{2} z_{2}+2 z_{1}^{2} z_{3} z_{4}-z_{1}^{2} z_{3}^{2} z_{4}-2 z_{3}^{2}+2 z_{1} z_{3}-4 z_{2} z_{3}+2 z_{4} z_{3} \\
& +2 z_{1} z_{4}+z_{1} z_{3}^{2}-z_{1}^{2} z_{3}+4 z_{3}^{2} z_{2}-z_{1}^{2} z_{3} z_{2} z_{4}+z_{1} z_{3}^{2} z_{2} z_{4} \\
& -2 x_{2} z_{3}^{2} z_{2} z_{4}-2 x_{2} z_{1}^{2}-2 x_{2} z_{1}^{2} z_{3} z_{2}+2 x_{2} z_{3}^{2}+3 x_{2} z_{1}^{2} z_{3}-x_{2} z_{2} \\
& +x_{2} z_{1}-3 x_{2} z_{2} z_{4} z_{1}+x_{2} z_{1} z_{3}^{2} z_{2} z_{4}-2 x_{2} z_{4} z_{3}-2 z_{3}^{2} z_{2} z \\
& +2 z_{1} z_{3}^{2} z_{4}-z_{3} x_{2}+z_{4} x_{2}+2 x_{2} z_{1} z_{2}+2 x_{2} z_{1}^{2} z_{2} z_{4}+3 z_{3} z_{2} z_{4} x_{2} \\
& \left.-x_{2} z_{1}^{2} z_{3} z_{2} z_{4}-x_{2} z_{1}^{2} z_{3}^{2} z_{4}-3 x_{2} z_{1} z_{3}^{2}+x_{2} z_{1}^{2} z_{3}^{2} z_{2}\right),
\end{aligned}
$$

and $x_{4}$ is obtained from the expression of $x_{3}$ by the exchanges $z_{1} \leftrightarrow z_{2}$ and $z_{3} \leftrightarrow z_{4}$. Therefore the map $f$ is two-to-one. 


\section{A system on $X(3,6)$ induced by $\mathcal{E}$ invariant un- der \#}

We have now the system

$$
\mathcal{E}(a, b, c), \quad a=5 b, c=4 b+1, b=b_{1}=\cdots=b_{4},
$$

with a parameter $b$ on $X(2,7)$ invariant under the involution \#. The push-down $f_{*} \mathcal{E}(a, b, c)$ of this system is a system defined on $X(3,6)$. Here we confess honestly that we still do not find a way to express the system $f_{*} \mathcal{E}(a, b, c)$ in $z$-variables with parameter $b$ in a reasonably compact form. Nevertheless when the system $\mathcal{E}(a, b, c)$ is non-sigular along the divisors $\left\{x_{j}=0\right\}$, that is, when $b=1 / 3$, we can explicitly find the coefficients of the system $f_{*} \mathcal{E}(a, b, c)$ and we obtain

Theorem 1 The system $\mathcal{E}(5 / 3,1 / 3,7 / 3)$, as a system on on $X(3,6)$, coincides with the system we found in [3], which has singularities along the divsor $\{D=0\}$.

Make the coordinate change $x \rightarrow z$ to transform the system $\mathcal{E}(a, b, c)$ into the form $\partial^{2} v / \partial z_{i} \partial z_{j}=\cdots$ and write the coefficients in terms of $x$. Though it is possible to express these coefficients in $z$, it is much easier to rewrite the coefficients of the system in [3] in termns of $x$. We then compare the coefficients of the two systems to find that they coincide.

\section{References}

[1] M. Hara, T. Sasaki And M. Yoshida, Tensor products of linear differential equations, Funkcial. Ekvac. 32(1989), 455-477.

[2] K. Matsumoto and T. Terasoma, Theta constants associated to cubic threefolds, in preparation, 2000.

[3] T. SASAKI AND M. Yoshida, The uniformizing differential equation of the complex hyperbolic structure on the moduli space of marked cubic surfaces, Proc. Japan Acad., 75(1999), 129 -133.

[4] M. Yoshida, Fuchsian Differential equations, Vieweg Verlag, 1987. 